\title{
Molecular docking based on construction of 4- $\alpha$-lanosterol demethylase (CYP51) as an active site of Madurella mycetomatis by homology modelling
}

Awad, TA 1 . Abd Algaffar, S.O1, Van de Sande, W.W ${ }^{3}$, Khalid SA2,

${ }^{1}$ King Faisal University, Hofuf, Saudi Arabia; ${ }^{2}$ Faculty of Pharmacy, University of Science \& Technology, Omdurman, Sudan, ${ }^{3}$ Erasmus MC, Department of Medical Microbiology and Infectious Diseases, Rotterdam, The Netherlands.

\section{Objectives}

Eumycetoma is a tropical neglected infectious disease causing large tumorous lesion on mainly the extremities. The current treatment consists of a combination of antifungal therapy with azoles and surgery. In order to predict which azoles, have the highest binding affinity to their target, a homology model based on the CYP51 target was constructed and its validity was tested by docking all the currently available azoles along with a set of synthetic compounds with established in vitro activities.

\section{Methods}

Madurella mycetomatis 14- $\alpha$-lanosterol demethylase (MmCYP51) protein sequence (GenBank:KXX80456.1), was built by comparative modelling method using the SWISS- MODEL server. BLAST feature of SWISS- MODEL was used to search for amino acid sequence in the template library (SMTL). A total of 50 templates showing identity to MmCYP51 targets ranged from $27.94 \%$ to $66.09 \%$ were found and the models are built based on the target-template alignment using ProMod3. Aspergillus fumigatus 14- $\alpha$-sterol demethylase (AfCYP51) was chosen PDB ID;4UYM, with sequence identity $66.09 \%$. and resolution of $2.55 \mathrm{~A}^{\circ}$. Conserved coordinates between target and template were copied to the model. Insertions and deletions were remodelled using a fragment library. Side chains were rebuilt and the geometry of the resulted model was regularized by using a force field. Ligands (heme and voriconazole) present in the template structure are transferred by homology to the model.

The global and pre-residue model was assesses using QMEAN scoring function and GMQE. The 3D superposition of target protein and template was carried out and RMSD for alignment determined. Docking was conducted with MOE docking module software. Compounds 3D structures were built with Chem3D software, then transferred and saved into MOE database. Energy for each compound was minimized by MMFF94x fore field. Finally, the 3D structures of prepared protein were saved as PDB file. A Gaussian Contact Surface was drawn around the binding site. Placement method used was Triangle Matcher. First scoring function was set to London $d G$ and the refinement to force filed. Docking started by retaining 30 poses and final refined poses were ranked by MM/GBV1 binding free energy.

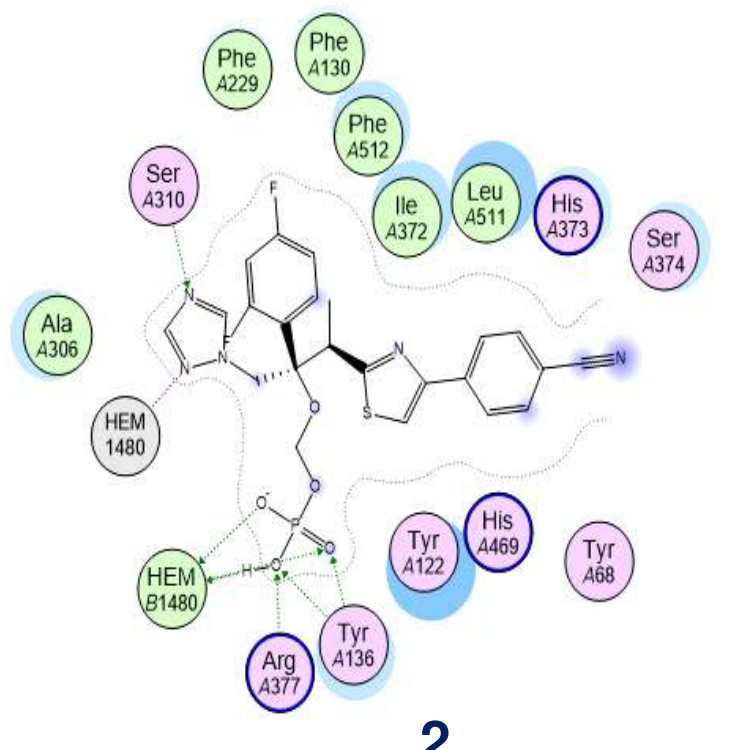

2

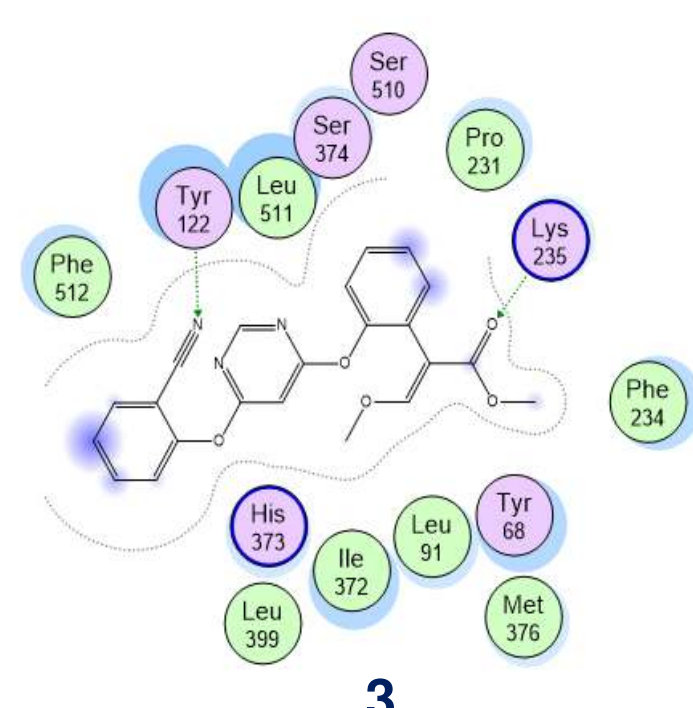

3
Figure 1 . The 2D interactions of fosravuconazole 2 and azoxystrobin 3 with MmCYP51

\section{Results}

The molecular docking was attempted using our constructed MmCYP51 homology model. Among the triazoles and a set of synthetic compounds subjected to in vitro screening against $M$. mycetomatis followed by docking on MmCYP51 homology model, fosravuconazole and azoxystrobin exhibited the best docking scores (Table 1). Docking revealed that these two compounds interacting with the developed model in a way that permits the phosphate group of fosravuconazole to form a potential hydrogen bonding, besides the nitrile and triazole moieties (Figure $1 \& 2$ ). This structural similarity between these two compounds provided further evidence of the role of a nitrile group in forming hydrogen bonding with the enzyme residues in a similar manner to the azoles (Figure 2).Furthermore, the remarkable difference in the docking values of the ravuconazole and fosravuconazole is apparently is due to the presence of an additional phosphate group in the later and its interaction by hydrogen bonding with Tyr A136, Arg A377 and heme 1480. It worth noting that eumycetoma is currently treated with itraconazole.

Table 1. In vitro screening and docking results of selected triazoles and azoxystrobin

\begin{tabular}{|l|c|c|}
\hline \multicolumn{1}{|c|}{ Compound } & $\begin{array}{c}\text { In vitro Result } \\
(\mathbf{I C 5 0} \boldsymbol{\mu M})\end{array}$ & $\begin{array}{c}\text { Docking Score } \\
\text { (Kcal/mol) }\end{array}$ \\
\hline Fosravuconazole & $-*$ & -20.37 \\
\hline Itraconazole & 0.17 & -17.69 \\
\hline Posaconazole & 0.18 & -16.91 \\
\hline Ravuconazole & 0.002 and 0.031 & -15.03 \\
\hline Voriconazole & 0.63 & -13.00 \\
\hline Azoxystrobin & 0.60 & -13.92 \\
\hline
\end{tabular}

*No in vitro data is available for the pro-drug fosravuconazole
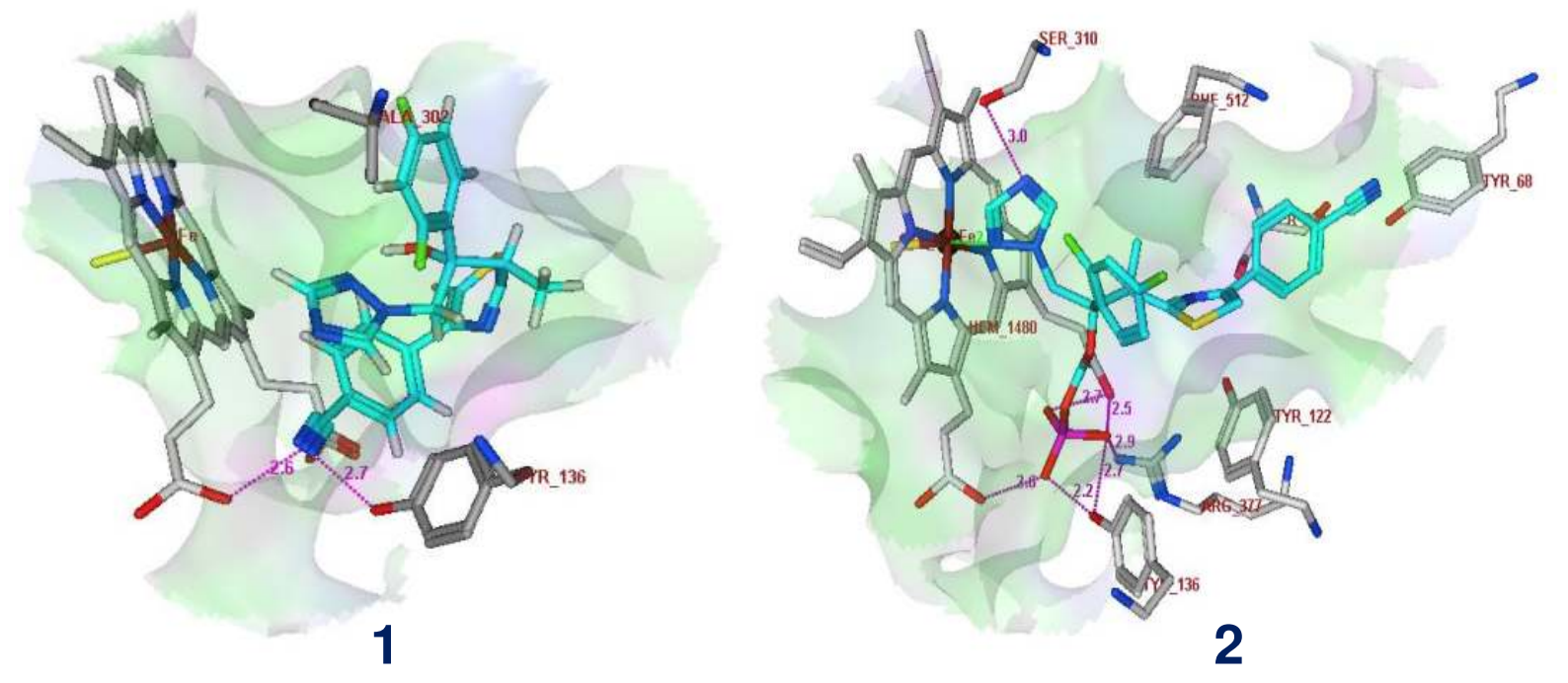

Figure 2. Ravuconazole 1 and fosravuconazole 2 3D interactions with MmCYP51

\section{Conclusion}

The constructed homology protein target seems to provide certain predictive value and correlation with the in vitro data. The pro-drug fosravuconazole emerged from this study as promising candidate for eumycetoma treatment, however, comprehensive research is required to discover other novel potent drugs.

\section{References}

1. Ahmed SA et al., Madurella mycetomatis is highly susceptible to ravuconazole, Plos Negl Trop Dis 8(6):e2942. 